\author{
G. Abitova \\ PhD, Candidate of Technical Sciences, Associate Professor of ICT \\ Educational Program \\ gulnara.abitova@astanait.edu.kz, orcid.org/0000-0003-3830-6905 \\ Astana IT University, Kazakhstan
}

\title{
MATHEMATICAL SIMULATION AND STUDY OF CONTROL STABILITY OF THE CHEMICAL-ENGINEERING PROCESSES IN INDUSTRY
}

\begin{abstract}
This work is considered to study analysis of dynamic system for simulation of the technological process under uncertainty and complexity. To study and simulate a complicated technology process the technical scheme of roasting in fluidized bed furnaces of polymetallic sulphides ores is carried out for consideration. The choice is justified by the fact that operation line producing polymetallic sulphide ores represents a complicated process, is characterized by a large number of transient processes, presence of process variables, and deviations from technical regimes. To study process characteristics of any system functioning employing mathematical methods the process should be formalized. This means, that an adequate mathematical model needs to be developed. The choice of mathematical model depends a lot on objects 'feature and its controllability as well as of technological scheme and complexity of processes. Chemical engineering processes are complicated physical and chemical systems. Substance flows, which are part of these systems, are, as a rule, multicomponent. Therefore, for the purpose of study and qualitative control over chemical-engineering processes it is essential to apply the method of mathematic simulation, based on system analysis strategy, analysis of its structure, mathematical formulation development and evaluation of unknown parameters. Controllability means that such system attribute as having control actions, which make it possible to transfer the system from a pre-set initial state to the required condition during a finite quantum of time. Therefore, the developed mathematical model of the process or control object should be controllable and stable.
\end{abstract}

Keywords: control system, mathematical model, stability, controllability, automation

\section{Introduction}

Control system design is one of the key tasks in the automation of all industry branches, including machine manufacturing, energy sector, electronics, chemical and biological, metallurgical, textile, transportation, robotics, aviation, and space systems, high-precision military systems, etc. It is widely known that most of the practical systems operate with some degree of parametric uncertainty resulting from poorly known parameters of controlled plants or unpredictable changes of their values in the process of operation [1].

Therefore, robust stability can be viewed as one of the outstanding issues in control theory, which is also of great practical interest. In general, the concept can be used to define the range of parameter variations that keep the system within stability boundaries. These boundaries are defined concerning to uncertain parameters of the controlled plant and can be affected by the settings of a control system. Known methods of control system design for objects with 
parametric uncertainty are usually based on determination of robust stability of systems with a given structure and with linear control laws or inertia-free (relay) characteristics [2].

They are usually not suitable for designing control systems with a wide range of robust stability when the range of parametric uncertainty or parametric drift is significant. Therefore, developing novel approaches to designing such systems is highly desirable. The results reported in [1, p.43] are obtained for dynamic systems that describe self-organizing processes in physical chemical, and biological systems. The models of these systems are presented in the form of structurally-stable mappings based on catastrophe theory and they can be used as universal models of development and self-organization in nature [3]. Therefore, it is of significant interest to consider great uncertainty of such systems and develop an automatic control system in the class of structurally stable mappings that uses the mathematical models reflecting complex behavior of the system with many possible stable solutions [2,4].

In this paper, we address the issues of modeling the robust control systems for dynamic linear objects with uncertain parameters. Special attention is paid to the selection of a control law in the class of structurally-stable mappings with one control factor $[5,6]$ that allows maximizing the range of robust stability and improves characteristics of the system.

\section{Materials and Methods}

To have an object-orientated and effective technological process implemented, it should be controlled. This means, that in the process of an engineering object functioning it is essential to have a continuous influence on operating members, to provide achievement of the required single result, in spite of external disturbances.

In other words, when saying system controllability, we understand such system attribute as having control actions, which make it possible to transfer the system from a pre-set initial state to the required condition during a finite quantum of time $[7,8]$.

Therefore, the developed mathematical model of the process or control object should be controllable. With this objection in mind let us consider our control object mathematical model and examine its controllability $[9,10]$.

Determination and Analysis Modelling Methods for Process

When simulating metallurgic processes and objects the most adequate approach is to choose the structural approach for mathematical model development. In this respect, for simulating sulphurisation process we will consider a molecular approach (as one of the structural approaches). Metallurgic processes and objects are described on this level as an aggregate of physical and chemical phenomena, particularly as an aggregate of chemical reactions.

For instance, the process of polymetallic sulphide ores concentrate oxidation in the first period at the molecular level can be described by the main chemical reaction of metal oxidation [11]:

$$
2 \mathrm{Me}+5 \mathrm{H}_{2} \mathrm{SO}_{4}=2 \mathrm{MeO}_{2} \cdot \mathrm{SO}_{3}+4 \mathrm{SO}_{2}+5 \mathrm{H}_{2} \mathrm{O} \text { (sulfurization) }
$$

and

$$
2 \mathrm{MeO}_{2} \cdot \mathrm{SO}_{3}+8 \mathrm{HCl}=2 \mathrm{MeCl}_{4}+\mathrm{H}_{2} \mathrm{SO}_{4}+3 \mathrm{H}_{2} \mathrm{O} \text { (dissolving) }
$$

where: $\mathrm{Me}$ - a concentrate of the first reagent (polymetallic sulphide ores)

$\mathrm{H}_{2} \mathrm{SO}_{4}$ and $\mathrm{HCl}$ - concentrates of the second reagents (sulfuric and perchloric acids)

$\mathrm{MeO}_{2} \cdot \mathrm{SO}_{3}$ - a concentrate of the oxidation reagent (sulphide oxidation)

$\mathrm{MeCl}_{4}$ - a concentrate of the final metal product (metal chloride).

The process of roasting in fluidized bed furnaces of polymetallic sulphide ores represents a complicated chemical-and-technological process, including different stages of raw material (sulfurization) chemical changes. The yielded product quality depends upon the valuable 
component recovery percent in the end product. That is why it is assumed justified to develop a mathematical model based on reactions of chemical kinetics.

Chemical kinetics is a science about chemical reactions proceeding in time behaviour and mechanism of these reactions. The main parameter here is some component formation rate. In metallurgy when modelling intricate chemical processes, normally, they are broken into separate stages of technological processes. Seeing that, we suggest considering modelling the main stages of sulfurization from metal concentrate, control over these stages is one of the critical targets.

The processing method of concentrate polymetallic sulphide ores with sulfurization usage enables to extract metal from a multiplex poly metallic raw material into commercial metal. This method of sulphide extraction includes the following operations:

- sulfurization (oxidation) of metal concentrate;

- metal pulp leaching and dressing;

- extraction and re-extraction of sulphide;

- primary metal settlement;

- sulphide melting.

Based on the experience and this technology research it is determined, that the main stages influencing the technological process and end product grade are sulfurization, and leaching. It means further technology of sulphide oxidation depends upon the control over these main stages and upon how they proceed.

Design and Analysis of Control System for the Process of Roasting in Fluidized Bed Furnaces of Polymetallic Sulphide Ores

As we discussed above, the mathematical model of chemical reactions in this type of process is described by mass balance equation of reagents $A$ (metal) и $B$ (oxidation reagent), considering the complicated nature of the process, with the presence of diffusion and mass transfer. Therefore, in this case, the task of control is: to maintain components concentrations $C_{A}$ and $C_{B}$ as closer to specified ones $C_{A s p}$ and $C_{B s p}$ (required), changing concentrations of reagents $A$ and $B$, i.e., $C_{A 1}$ and $C_{B 1}$ [12].

To design the control system for considering process, we simplify the equations, introducing relative coordinates, and write the following expressions:

$$
d_{1}=\left(k_{1} * V\right) / F \quad \text { and } \quad d_{3}=\left(k_{3} * V\right) / F
$$

where: $d_{1}$ - a concentrate of the first component in reactions

$d_{2}$ - a concentrate of the second component in reactions.

$$
t=\left(t^{\prime} * F\right) / V
$$

where: $t$ - period (time) needed for oxidation process of the metals.

$$
\begin{aligned}
& x_{1}=\frac{C_{A}}{C_{\text {Aran }}} \quad \text { and } \quad x_{2}=\frac{C_{B}}{C_{\text {Aran }}} \\
& u_{1}=\frac{C_{A 1}}{C_{\text {Aran }}} \quad \text { and } \quad u_{2}=\frac{C_{B}}{C_{\text {Aran }}}
\end{aligned}
$$

where: $C_{A r a n}$ - some chosen at random concentration value $C_{A}$

$d_{1}, d_{3}$ - coefficients for corresponding steps.

Then, after changing variables and parameters, the designing control system of our equations in these coordinates will give by:

$$
\frac{d x_{1}}{d t}=-\left(1+d_{1}\right) * x_{1}+u_{1} ; \quad x_{1}(0)=x_{10}
$$




$$
\frac{d x_{2}}{d t}=d_{1} * x_{1}-\left(1+d_{3}\right) * x_{2}+u_{2} ; \quad x_{12}(0)=x_{20}
$$

Study of Controllability and Stability of the Control System of Process: First Task

In this case, the control target of regulating is defined this way: it is essential to maintain lout coming concentrations $x_{1}$ and $x_{2}$ on specified levels, changing incoming concentrations $u_{1}$ and $u_{2}$.

Let us put the control system equation in vector-matrix form:

$$
\begin{gathered}
\frac{d}{d t}\left[\begin{array}{l}
x_{1} \\
x_{2}
\end{array}\right]=\left[\begin{array}{cc}
-\left(1+d_{1}\right) & 0 \\
d_{1} & -\left(1+d_{3}\right)
\end{array}\right] *\left[\begin{array}{l}
x_{1} \\
x_{2}
\end{array}\right]+\left[\begin{array}{ll}
1 & 0 \\
0 & 1
\end{array}\right] * u \\
{\left[\begin{array}{l}
x_{1}(0) \\
x_{2}(0)
\end{array}\right]=\left[\begin{array}{l}
x_{10} \\
x_{20}
\end{array}\right] \quad \text { and } x_{10}=0, \quad x_{20}=0} \\
y(t)=\left[\begin{array}{ll}
1 & 1
\end{array}\right] *\left[\begin{array}{l}
x_{1} \\
x_{2}
\end{array}\right] \quad \text { and } u(t)=\left[\begin{array}{ll}
u_{1} & u_{2}
\end{array}\right]
\end{gathered}
$$

Once the model under consideration is rectilinear, it can be represented as an equation system in space of states (in the time domain). In standard form, the system is given by:

$$
\begin{gathered}
A=\left[\begin{array}{cc}
-\left(1+d_{1}\right) & 0 \\
d_{1} & -\left(1+d_{3}\right)
\end{array}\right] \\
B=\left[\begin{array}{ll}
1 & 0 \\
0 & 1
\end{array}\right] \quad C=\left[\begin{array}{ll}
1 & 1
\end{array}\right] \text { and } D=0
\end{gathered}
$$

Analysis of Controllability for Control System of Process

It is very important when we design a control system, to know, that this system has controllability [13]. The system is controllable if it has such control as a $u(t)$, which can transfer a control system from her initial state $x_{0}$ to any state $x_{d}$ within final time.

Therefore, for current systems with matrixes $A$ and $B$ the controllability conditions are what control system will be full controllability, when rang of a matrix of controllability $L c$ equal $n(L c=n)$, where:

$$
L c=\left[B: A * B: A^{2}: \cdots: A^{n-1} * B\right]
$$

In our case, to examine system controllability we make a controllability matrix for our case of the process.

Respectively controllability matrix $L$ is given by:

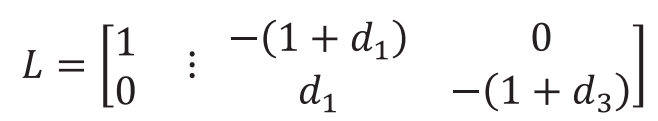

In this case the, controllability matrix rank $L$ is also equal to two $(n=2)$, and this ensures the process controllability with one control action, though the quality of control will worsen. Thus, we can say, that our designed control system for the tellurium oxidation process in the common case can be controllable when we take a right regulation unit for keeping given regimes of technological process. 


\section{Results and Discussion}

There is not sufficient possibility to control both parameters and variables in real technological processes and situations. Consequently, analysis and study of the other condition for the control system is needed.The stability conditions for the control plan are stronger that construability of the system.

The stability condition is in that, all of the instability modes and eigenvectors of the control system can be made stable by regulation impact in the closed-loop system.

Let us consider a case of the control system of the oxidation process of polymetallic sulphide ores for the linear regulator with constant coefficients, which are given in state-space by the expression:

$$
u(t)=-K * x(t)
$$

where: $K$ - is $\left(m^{*} n\right)$ matrix of closed loop system.

System (16) with this regulator can be show in follow form:

$$
\frac{d x}{d t}=(A-B * K) * x
$$

It is following from above form, that stability of the system can be in that case, when we can choose a matrix of the closed loop system $K$. In this way, all of the eigenvectors of the matrix $\left(A-B^{*} K\right)$ have negative real numbers.

If it is a case with control regulation by out coming:

$$
u(t)=-K * y(t)
$$

then a system will be given in the next form:

$$
\frac{d x}{d t}=(A-B * K * C) * x
$$

In this case, stability is proved by choosing such matrix $K$, when a real eigenvector of the matrix $(A-K * B * C)$ will be negative.

Numerical Experiment Simulation of the Control System of Technological process

The designed control system for the technological process of roasting in fluidized bed furnaces of polymetallic sulphide ores and its controllable can be demonstrated by numerical simulations using computer software and modern technology of programming.

Let us consider this model, using the actual examples of technological processes, having modelled it in MATLAB and Simulink. Assuming, that system parameters have the following values (Table 1) (as an example):

Table 1. System parameters and their values

\begin{tabular}{|c|c|c|c|c|}
\hline Number of Experiments & \multicolumn{4}{|c|}{ Values of Parameters } \\
\hline Parameters & $\boldsymbol{k}$ & $\boldsymbol{x}$ & $\boldsymbol{u}$ & $\boldsymbol{d}$ \\
\hline 1 & $k 1=1$ & $x 1=A$ & $u 1=-k 1 *_{x} 1$ & $D 1=3$ \\
\hline 2 & $k 2=2$ & $x 2=B$ & $u 2=-k 2 * x 2$ & $D 2=1$ \\
\hline
\end{tabular}

Here the $A$ is a concentrate of polymetallic sulphide ores and $B$ is the concentration of oxidation reagent.

The mathematical model can be written in the form of equation for state space:

$$
\frac{d x}{d t}=A * x+B * u \quad \text { and } \quad y=C * x
$$


In this case, matrices of $A$ and $B$ will be written in the form of:

$$
A=\left[\begin{array}{rr}
-4 & 0 \\
3 & -2
\end{array}\right] \quad B=\left[\begin{array}{ll}
1 & 0 \\
0 & 1
\end{array}\right] \quad C=\left[\begin{array}{ll}
1 & 1
\end{array}\right] \quad D=0
$$

The model of the process of roasting in fluidized bed furnaces of polymetallic sulphide ores and the controller are set in such a way that, in the linearized form, the following parameters of the system are used in five different numerical simulations using MATLAB (Table 2). Having made numerical experiments in MATLAB, we get the following results and diagrams (Fig.1. Fig.3):

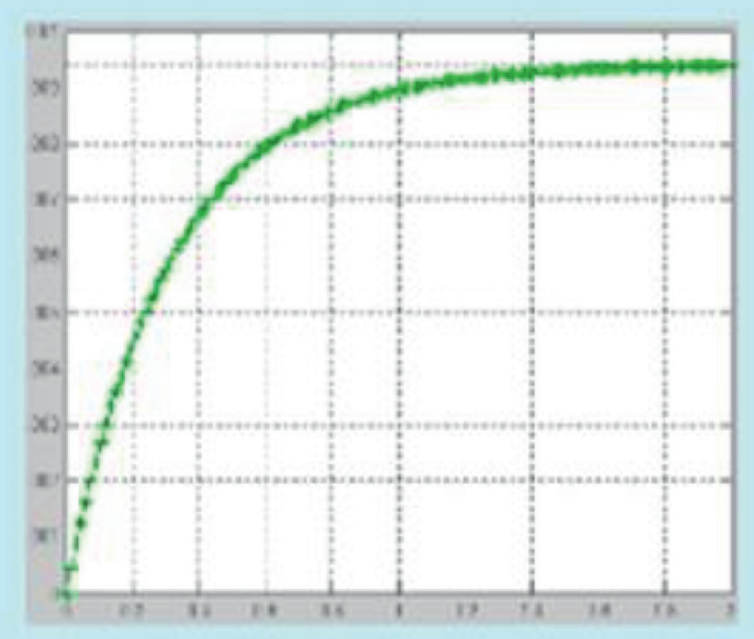

a) $k 1=1 \quad k 2=2$

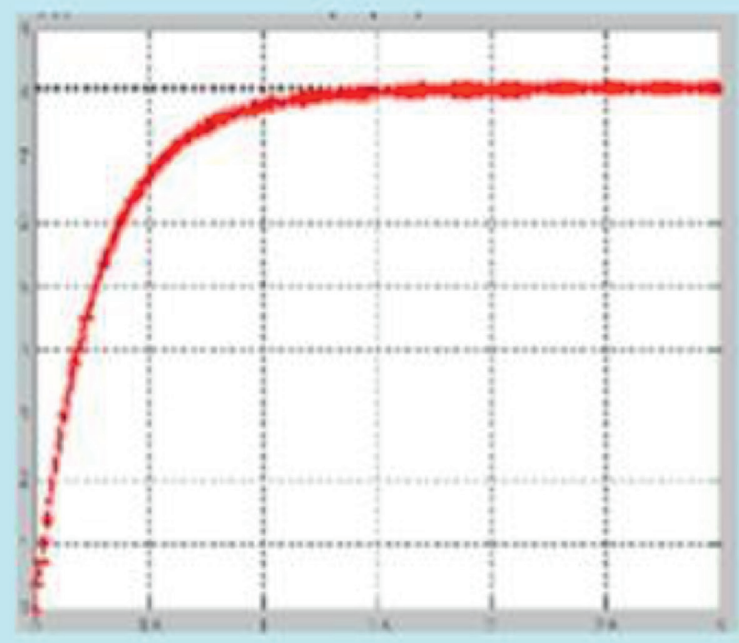

b) $k 1=1 \quad k 2=-2$

Fig. 1. Numerical simulation results: Experiment 1-2

The second experimental results are representing below:

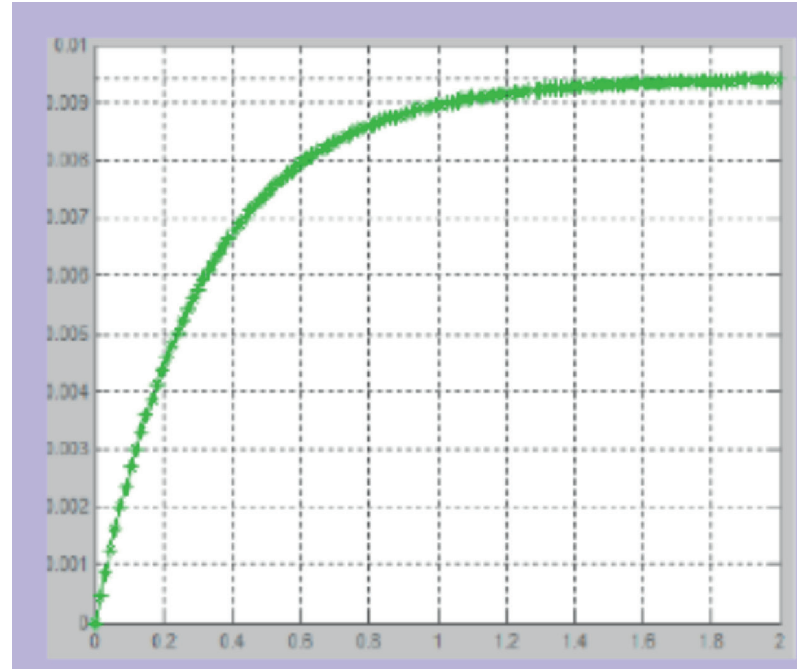

a) $k 1=1 \quad k 2=-1.5$

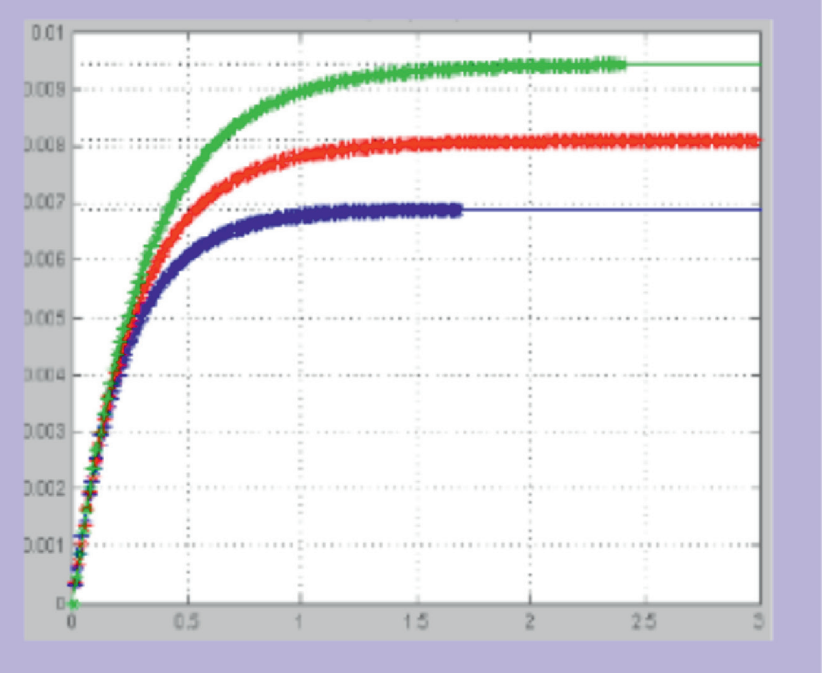

b) $k 1=-1 \quad k 2=2$

Fig. 2. Numerical simulation results: Experiment 3-4 
The waveforms presented in Fig.1-Fig.2 show stable performance for a different set of parameters and converge to equilibrium points.

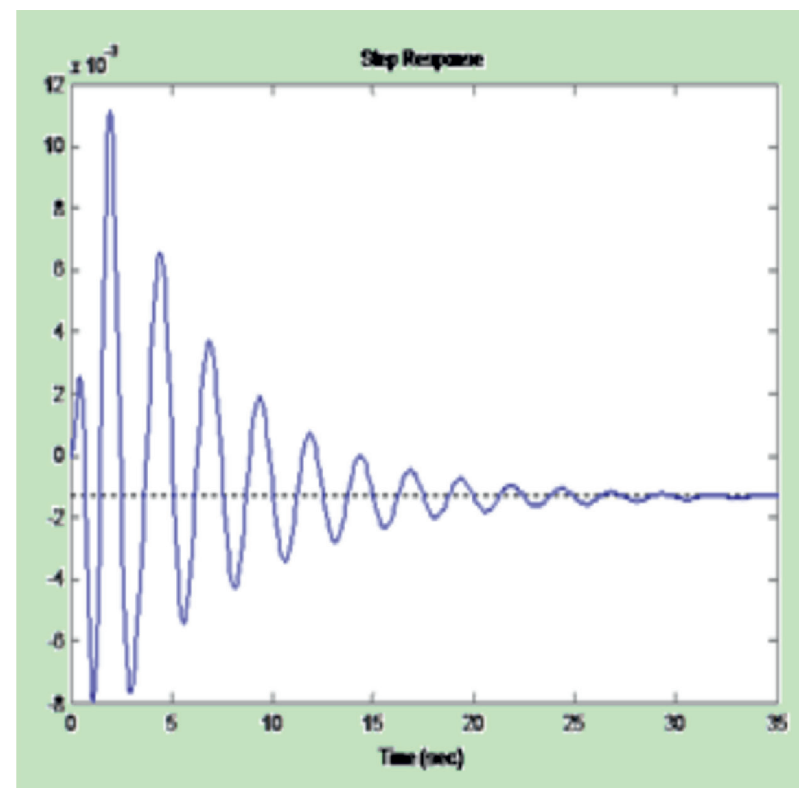

a) $k 1=-1 \quad k 2=-2$

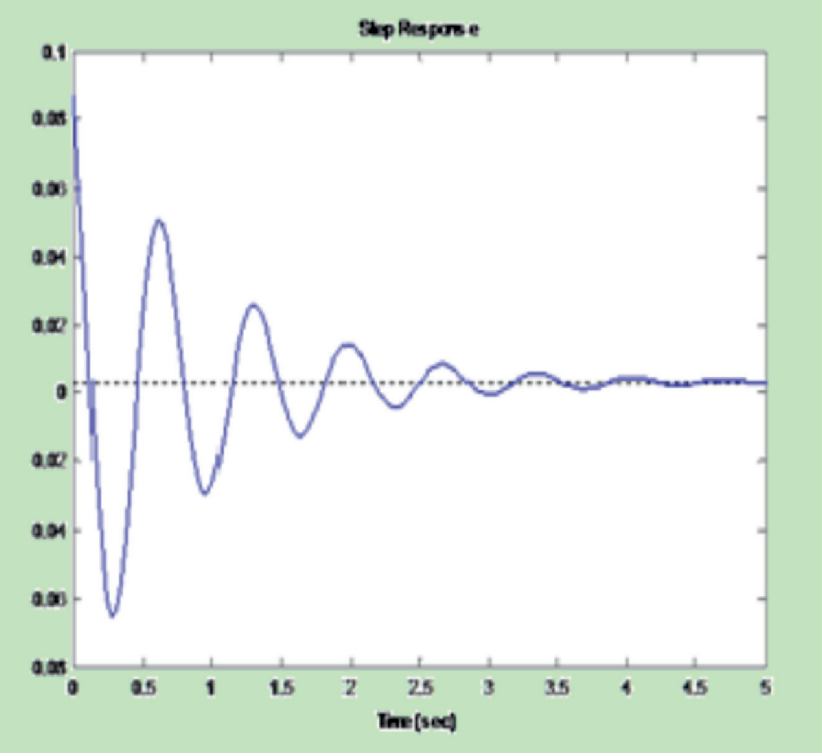

b) $k 1=2 \quad k 2=3$

Fig. 3. Numerical simulation results: Experiment 5-6

This set of parameters results in an asymptotically stable system whose convergence process is shown in Fig.3.

The waveforms in Fig.1 and Fig.2 are of particular interest since they demonstrate stability. The results presented above illustrate that system can converge an equilibrium point even if the dynamics of the controlled process suddenly changes.

However, in practice, it is not always possible to control simultaneously all the parameters, and variables. That is why the considered above case is an example of the most idealized option (Table 2).

Table 2. The values of parameters for numerical simulations

\begin{tabular}{|c|c|c|c|}
\hline Number of Experiments & \multicolumn{3}{|c|}{ Value of Parameters and Equations } \\
\hline $\boldsymbol{n}$ & $\boldsymbol{k} \mathbf{k}$ & $\boldsymbol{k}$ & $\boldsymbol{u}=\boldsymbol{u} \mathbf{1}+\boldsymbol{u} \boldsymbol{2}$ \\
\hline a) & 1 & 2 & $u=-1 * x 1-2 * x 2$ \\
\hline b) & -1 & -2 & $u=1 * x 1+2 * x 2$ \\
\hline c) & -1 & 2 & $u=1 * x 1-2 * x 2$ \\
\hline d) & 1 & -2 & $u=-1 * x 1+2 * x 2$ \\
\hline e) & 2 & 3 & $u=-1 * x 1-2 * x 2$ \\
\hline k) & 1 & -1.5 & $u=-1 * x 1-1.5 * x 2$ \\
\hline
\end{tabular}

Numerical Experiment Simulation of the Control System of the Technological Process

The designed control system for the real technological process of tellurium oxidation and its controllable can be demonstrated by numerical simulations using computer software and modern technology of programming.

This model can be considered for second case, using the actual example of technological processes, having modelled it in MATLAB and Simulink.

The pursued numerical experiments are given below (Table 3 ). 
Table 3. Values of parameters for numerical experiments

\begin{tabular}{|c|c|c|c|}
\hline Number of Experiments & \multicolumn{3}{|c|}{ Value of Parameters and Equations } \\
\hline $\boldsymbol{N}$ & $\boldsymbol{k} \mathbf{k}$ & 0 & $\boldsymbol{u}=\boldsymbol{u} \mathbf{1}$ \\
\hline a) & 3 & 0 & $u=-3 * x 1$ \\
\hline b) & 1 & $u=1 * x 1$ \\
\hline
\end{tabular}

Experimental results of thetechnological process using Matlab are given below in the Fig.4 -Fig.5.

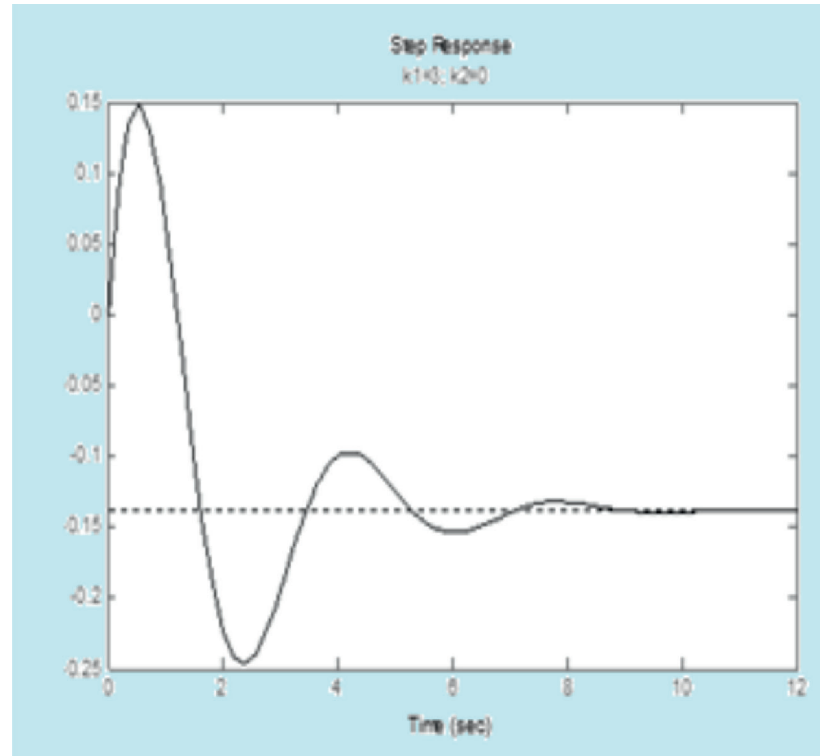

a) $k 1=3 \quad k 2=0$

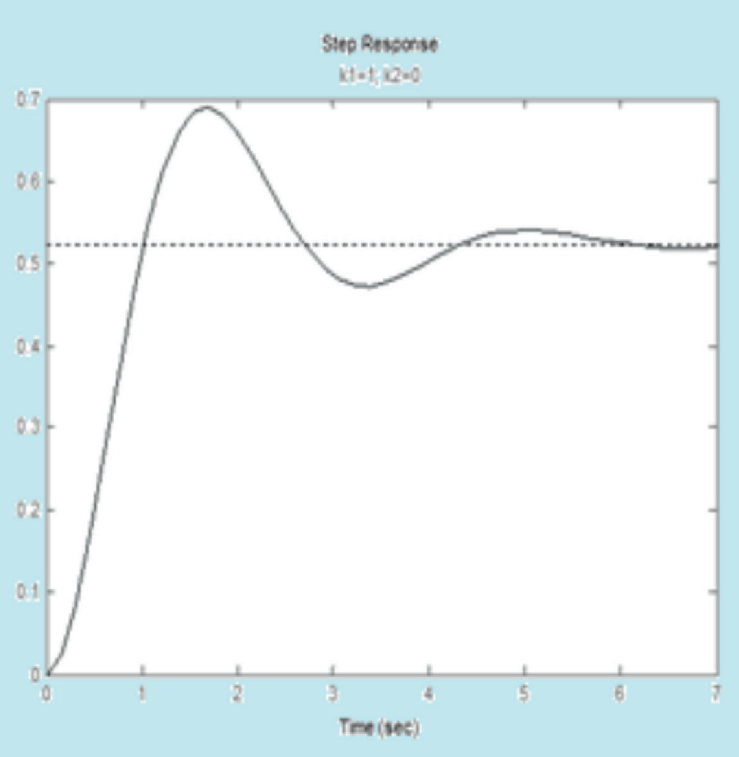

b) $k 1=1 \quad k 2=0$

Fig. 4. Numerical simulation results: Experiment 7-8

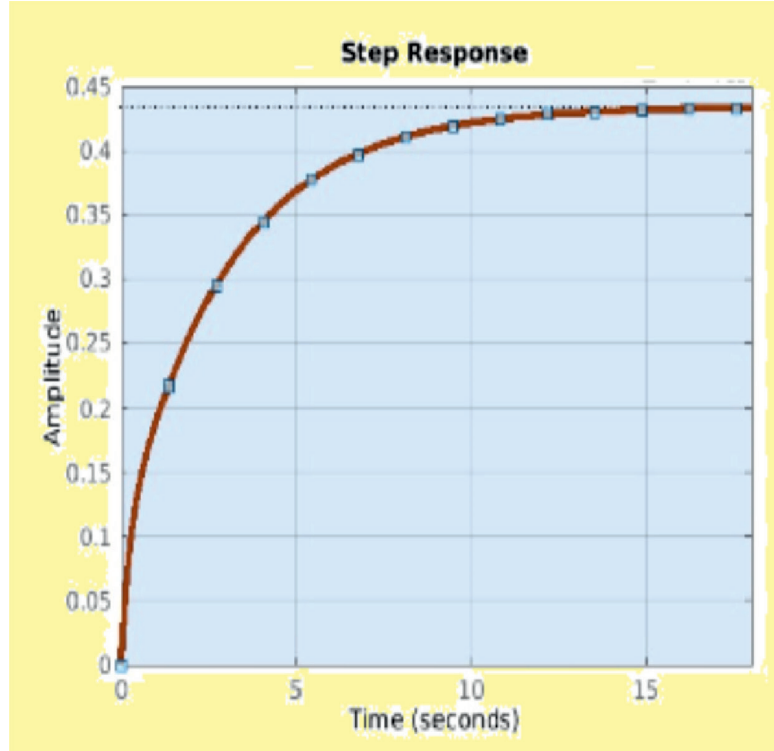

a) $k 1=2 \quad k 2=3$

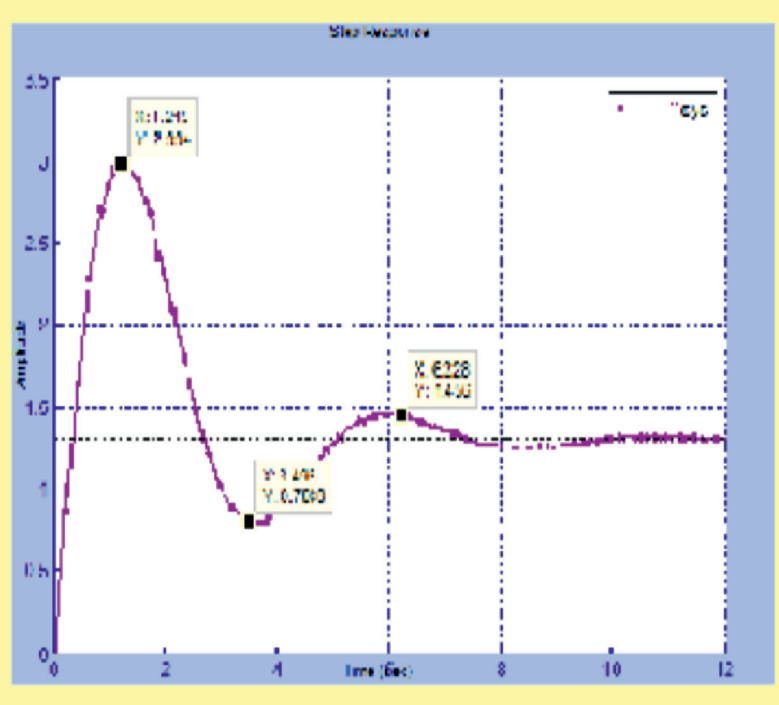

b) $k 1=1 \quad k 2=0$

Fig. 5. Numerical simulation results: Experiment 9-10 
The waveforms presented in Fig.4 show stable performance for a different set of parameters with less quality and converge to equilibrium points. In this case, this ensures the process controllability with one control action, though the quality of control will worsen.

Thus, for the second model the waveforms in these are of particular interest, since they demonstrate stability. The results presented above illustrate that it is possible for the system to converge to an equilibrium point even if the dynamics of the controlled process suddenly changes. Though in practice, it is not always possible to control simultaneously all the parameters, and variables.

In real technological processes and situations, we don't have a possibility to control both parameters and variables. Therefore, it needs to analyze and study the other condition for the control system with the modeling of technological processes. It is the stability conditions for the contol plant, that are more stronger than controbaleof the system.

In the case of model 1 , in practice it means, that, though both out coming concentrations of substances cannot be controlled only by changing $B$ substance consumption. Concentration $A$ is varying persistently and approaches to steady-state irrespective of control. It means, the regulator for the system can be arranged, in spite of the fact, that the system is not fully controlled. Thus, the control object is fully controlled towards controls A, but is not fully controlled towards control B.

For the second model, the waveforms in these are of particular interest since they demonstrate robust stability and controllability. The results presented above illustrate that it is possible for the system to converge to an equilibrium point even if the dynamics of the controlled process suddenly changes.

\section{Conclusion}

Modern control methodologies are capable of increasing the productivity of industrial cycles by means of automatic control, monitoring, and management systems. The components used for such control and monitoring tasks, including modern different sensors and devices, are usually tightly integrated with automatic systems that control the entire technological process. This approach facilitates the reduction of expenses used to service the main assets, allows increasing output productivity, reducing downtime, and finally increases the controllability and stability of complex technological processes of industry.

At the same time for the purpose of studying and qualitative control over chemicalengineering, it is essential to apply the method of mathematic simulation, based on system analysis strategy, qualitative analysis of its structure, mathematical formulation development,and evaluation of unknown parameters. Therefore,the study of control systems and modeling of technological processes with robust stability is one of the most important directions for enhancement, optimization, and intensification of the production facilities. The work in this part of the research briefly describes an approach to assure robustness of a dynamic system. Its primary focus is on robust stability, not robust performance, in the presence of parameter variations and model uncertainties. Based on our analysis, the paper presents the conditions for converging to an equilibrium point and explains how to implement a controller to achieve robust stability for the technological process of roasting in fluidized bed furnaces of polymetallic sulphide ores.

In a number of applications where a system is characterized by nonlinear and timevarying behavior, the issue of stability and performance becomes very prominent. It is further complicated by the fact that when a mathematical model is used as part of the control strategy, it is typically assumed to closely match the real process. However, the process-model mismatch often results in sub-standard performance or even stability issues. The latter can stem from 
improper controller gains that were chosen based on the assumed model of the controlled process.

The stability condition is in that, all of instability modes and eigenvectors of the control system can be made stable by regulation impact in the closed-loop system. Considered a simulation of the technological process (process of roasting in fluidized bed furnaces of polymetallic sulphide ores), we found conditions for the stability of the system, the radius and transients. Thus, our robust stability study is specifying constraints on the change control system parameters that preserve stability. These limits are determined by the region of stability in an uncertain and are selected. Using these methods of modelling and designing of thecontrol system and results of these studies we can design more effective control systems for the technological process and tobtain a product with high quality.

\section{References}

1. Polyak B., \& Scherbakov P. (2002). Robust Stability and Control. Publisher: Nauka, Moscow.

2. Abitova Gulnara (2017). Control Systems and Industrial Automation of Rare Metals Production. In: Monography. Scientific Publication. Lap Lambert Academy Publishing. OmniScriptum GmbH \& Co. KG - Beau Bassin, Germany, 161 p.

3. G. Abitova, V. Nikulin, M. Beisenbi (2010). Design of a Control System with High Robust Stability Characteristics. The 3rd International Congress on Ultra Modern Telecommunications and Control Systems. The Proceedings of the ICUMT2011, Budapest, Hungary.

4. Xiaoping Zh., Sun Jiahui, \& Cheng Yuan (2010). «Analysis of crowd jam in public buildings based on cusp-catastrophe theory» Building and Environment, 45, Issue 8, Publisher: Elsevier Ltd, 1755-1761.

5. Nikolas G., \& Prigozhin I. (1990). Complex Discovery. Publisher: Mir, Moscow.

6. Wang, S. C., She, X. S., Li, Y., \& Mu, P. (2010). Stability analysis of rock slope based on cusp catastrophe theory. Journal of traffic and transportation engineering (Xian, Shaanxi), 10(3), 23-27.

7. Alexandrov, \& Zhabko A. (2012). On Asymptotic Stability of Solutions to Systems of Nonlinear Differential Equations with Time-Delay. Izvestiya VUZov, Mathematics, (5), 3-12.

8. Zhang, Y., Fidan, B., \& loannou, P. A. (2003). Backstepping control of linear time-varying systems with known and unknown parameters. IEEE Transactions on Automatic Control, 48(11), 1908-1925.

9. Abitova, G., \& Nikulin, V. (2012). Increasing product quality by implementation of a complex automation system for industrial processes. In Complex Systems Design \& Management (pp. 305316). Springer, Berlin, Heidelberg.

10. Abitova, G. Research and development of control system for process with increased potential robust stability at complex automation of production of rare metals (Doctoral dissertation, doctoral dissertation, PhD. KazNTU. - Almaty. - 2013. - 149 p).

11. Kenzhebaeva, T.S., Orazbayev, B.B., Abitova, G.A., Orazbayeva, K.N., \& Spichak, Y.V. (2017). Study and Design of Mathematical Models for Chemical-Technological Systems under Conditions of Uncertainty Based on the System Analysis. In Proceedings of the International Conference on Industrial Engineering and Operations Management (pp. 776-790).

12. Abitova, G.A. (2006). Совершенствование процесса извлечения теллура из плавов свинцово-цинкового производства с применением новых информационных технологий.: diss. cand. tech. scien. Institute of Metallurgy and Enriched, NAS MES of RK. Almaty. - 2006. - 148 p.

13. Morse, A. S. (1992). High-order parameter tuners for the adaptive control of linear and nonlinear systems. In Systems, models and feedback: Theory and Applications (pp. 339-364). Birkhäuser, Boston, MA. 